\title{
DIRECT N-POINT DCT COMPUTATION FROM THREE ADJACENT N/3-POINT DCT COEFFICIENTS
}

\author{
Soo-Chang Pei and Meng-Ping Kao \\ Department of Electrical Engineering \\ National Taiwan University \\ Taipei, Taiwan 10617, ROC \\ E-mail: pei@cc.ec.ntu.edu.tw ， Fax: 886-2-23671909
}

\begin{abstract}
An efficient method for computing a length- $N$ DCT given three consecutive length- $N / 3$ DC'ls is proposed. This method differs from previous ones in that it reduces considerable arithmetic operations and uses only length$N / 3$ DCTs instead of length- $N$ DCTs. We also find its great applications in fractional scaling of a DC'-based image by the factor of $N / 2^{\alpha} 3^{\beta}$. This would be very usclul in HDTV standard, whose display size is usually $16: 9$. The comparison with conventional methods is provided in this paper.
\end{abstract}

\section{INTRODUCTION}

Since "Discrete Cosine Transform (DCT)" was first introduced in 1974, it has been widely used in various fields among digital signal processing (DSP). For example, it is the foundation stone of many prevailing image and video compression techniques, such as JPEG, MPEGs and H.26x. Moreover, most of the images and videos nowadays are stored in the DCT domain through the above techniques. The question, however, arises that how can we directly manipulate or process such a compressed media stored in DCT domain.

To avoid unnecessary computations in decompression and recompression, a number of algorithms have been proposed to complete the operations directly in the DCT domain. Among the various operations, scaling of an image, i.e. interpolation or decimation is probably the most common ones we might encounter in relevant applications. For example, Park [4] has proposed an algorithm using the symmetric convolution property of DCT. Zhao [5] utilizes the relationship between linear transforms in spatial domain and those in DCT domain to develop an alternative approach

In compressed image or video domain, it is necessary to construct a long DCT sequence from several short DCT sequences or to decompose a long DCT sequence into several short DC'T sequences. Since Skodras [I] has proposed a corresponding method for the case of two sequences, we now focus on the case of three consecutive sequences in this paper. Combining with Skodras's method, many DCT domain operations, such as fractional scaling by the lactor of $N / 2^{\alpha} 3^{p}$, are now realizable in a simple and efficient way.

\section{PROPOSED APPROACH}

I.et us readdress the problem as follows. Given three sequences of the same length $N / 3$, i.e. $A_{k}, B_{k}$ and $C_{k}$, which are the DCT coefficients of three consecutive sequences $a_{n}, b_{n}$ and $c_{n}$ respectively. Suppose that $a_{n}$, $b_{n}$ and $c_{n}$ together form the sequence $x_{n}$ of length $N$, i.e. $a_{n}=x_{n} \quad, \quad b_{n}=x_{n+(N / 3)} \quad, \quad c_{n}=x_{n+(2 N / 3)} \quad$ for $n=0,1, \cdots, N / 3-1$, how can we find an efficient way to compute $X_{k}$ from $A_{k}, B_{k}$ and $C_{k}$ ?

The traditional way to solve this problem is depicted in Fig. 1. According to this scheme, three $N / 3$-point DCTs are required followed by a $N$-point DCT. However, even if the fast DCT structure [3] is used, the computation complexity is approximately $N \log _{2} N$ proportional to $N$, which means the $N$-point DCT in Fig.l will always dominate the whole computational load.

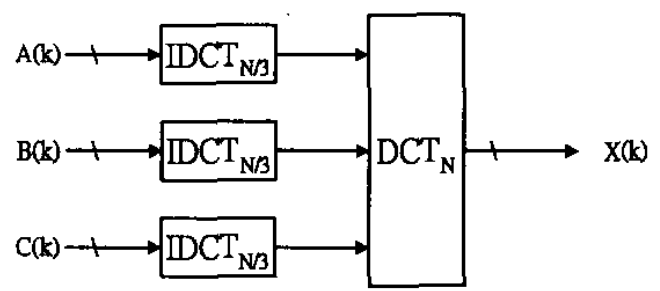

Fig. 1 Schematic representation of the traditional approach

In order to modify the traditional approach, we propose a new structure based on DCT decomposition to avoid the $N$-point DCT computation. In other words, we 
use only $N / 3$-point DCTs to find $X_{k}$, which is much more efficient than before.

Now we get straight to investigate the key concept of this new structure. First of all, the normalized forward DCT-II of length- $N$ sequence $x_{n}$ is given as follows,

$$
x_{k}=\sqrt{\frac{2}{N}} \varepsilon_{k} \sum_{n=0}^{N-k} x_{n} \cos \frac{(2 n+1) k \pi}{2 N}, k=0,1, \cdots, N-1
$$

and the corresponding inverse DCT is

$x_{n}=\sqrt{\frac{2}{N}} \sum_{k=0}^{N-1} c_{k} X_{k} \cos \frac{(2 n+1) k \pi}{2 N}, n=0,1, \cdots, N-1$

where

$$
\varepsilon_{k}=\left\{\begin{array}{c}
1 / \sqrt{2}, \text { for } k=0 \\
1, \text { for } k \neq 0
\end{array}\right.
$$

Note that $s_{3 k}=\varepsilon_{k}$ and $\varepsilon_{3 k+1}=\varepsilon_{3 k+2}=1$. We now decompose $I_{k}$ to three equal-length parts, $I_{3 k}$, $X_{3 k+1}+X_{3 k-1}$ and $X_{3 k+2}+X_{3 k-2}$ separably.

\section{1. $\mathrm{X}_{3 \mathrm{k}}$ COEFFICIENTS CALCULATION}

$$
\begin{aligned}
& X_{3 k}=\sqrt{\frac{2}{N}} \varepsilon_{3 k} \sum_{n=0}^{N-1} x_{n} \cos \frac{(2 n+1) 3 k \pi}{2 N} \\
& =\sqrt{\frac{2}{N}} \varepsilon_{k}\left\{\sum_{n=0}^{(N / 3)-1} a_{n} \cos \frac{(2 n+1) k \pi}{2(N / 3)}\right. \\
& +\sum_{n=\{(N / 3)-1}^{0} b_{N / 3-1-n} \cos \frac{(2(N / 3-1-n+N / 3)+1) k \pi}{2(N / 3)} \\
& \left.+\sum_{n=0}^{(N / 3)-1} c_{n} \cos \left\{\frac{(2 n+1) k \pi}{2(N / 3)}+2 k \pi\right)\right\} \\
& =\sqrt{\frac{1}{3}} \sqrt{\frac{2}{N / 3}} \varepsilon_{k}\left\{\sum_{n=0}^{(N / 3)-1} a_{n} \cos \frac{(2 n+1) k \pi}{2(N / 3)}\right. \\
& \left.+\sum_{n=0}^{(N / 3)-1} b_{n}^{\prime} \cos \frac{(2 n+1) k \pi}{2(N / 3)}+\sum_{n=0}^{(N / 3)-1} c_{n} \cos \frac{(2 n+1) k \pi}{2(N / 3)}\right\} \\
& =\sqrt{\frac{1}{3}}\left\{A_{k}+B_{k}^{\prime}+C_{k}\right\}, k=0,1, \cdots, \frac{N}{3}-1
\end{aligned}
$$

where $a_{n}=x_{n}, b_{n}=x_{n+(N / 3)}, c_{n}=x_{n+(2 N / 3)}$, and

$$
\begin{aligned}
b_{n}^{\prime}=b_{(N / 3)-1-n} & =x_{(2 N / 3)-1-n)} \\
& \text { for } n=0,1, \cdots,(N / 3)-1
\end{aligned}
$$

As a consequence,

$$
\begin{array}{r}
B_{k}^{\prime}=D C T_{N / 3}\left\{b_{n}^{\prime}\right\}=D C T_{N / 3}\left\{b_{(N / 3)-1-n}\right\}=(-1)^{k} B_{k}, \\
\text { for } k=0,1, \cdots,(N / 3)-1
\end{array}
$$

\section{2. $X_{3 k+1}+X_{3 k-1}$ COEFFICIENTS CALCULATION}

$$
\begin{aligned}
& X_{3 k+1}+X_{3 k-1} \\
&= \sqrt{\frac{2}{N}} \sum_{n=0}^{N-1} x_{n}\left\{\cos \frac{(2 n+1)(3 k+1) \pi}{2 N} \varepsilon_{3 k+1}\right. \\
&\left.+\cos \frac{(2 n+1)(3 k-1) \pi}{2 N} \varepsilon_{3 k-1}\right\} \\
&= \sqrt{\frac{2}{N} \sum_{n=0}^{N-1} x_{n} 2 \cos \frac{(2 n+1) \pi}{2 N} \cos \frac{(2 n+1) 3 k \pi}{2 N}} \\
&= 2 \sqrt{\frac{2}{N}}\left\{\sum_{n=0}^{(N / 3)-1}\left(a_{n} \cos \frac{(2 n+1) \pi}{2 N}\right) \cos \frac{(2 n+1) k \pi}{2(N / 3)}\right. \\
&+\sum_{n=0}^{N N / 3 k-1}\left(b_{n} \cos \frac{(2(n+N / 3)+1) \pi}{2 N}\right) \cos \frac{(2(n+N / 3)+1) k \pi}{2(N / 3)} \\
&\left.+\sum_{n=0}^{\{N / 3)-2}\left(c_{n} \cos \frac{(2(n+2 N / 3)+1) \pi}{2 N}\right) \cos \frac{(2(n+2 N / 3)+1) k \pi}{2(N / 3)}\right\}
\end{aligned}
$$

Assume that

$$
\theta_{n}=\frac{(2 n+1) \pi}{2 N}
$$

then

$$
\begin{aligned}
& 2 \sqrt{\frac{2}{N}} \frac{1}{\varepsilon_{k}} \sqrt{\frac{N / 3}{2}}\left\{\sqrt{\frac{2}{N / 3}} \varepsilon_{k} \sum_{n=0}^{(N / 3)-1}\left(a_{n} \cos \theta_{n}\right) \cos \frac{(2 n+1) k \pi}{2(N / 3)}\right. \\
& +\sqrt{\frac{2}{N / 3}} \varepsilon_{k} \sum_{n=0}^{(N / 3)-1}\left(b_{n}^{\prime} \cos \left(\frac{(2 n+1) \pi}{2 N}-\frac{2 \pi}{3}\right)\right\} \cos \frac{(2 n+1) k \pi}{2(N / 3)} \\
& \left.+\sqrt{\frac{2}{N / 3}} \varepsilon_{k} \sum_{n=0}^{(N / 3)-1}\left(c_{n} \cos \left(\frac{(2 n+1) \pi}{2 N}+\frac{2 \pi}{3}\right)\right) \cos \frac{(2 n+1) k \pi}{2(N / 3)}\right\} \\
& =\sqrt{\frac{1}{3} \frac{1}{\varepsilon_{k}}}\left\{D C T_{N / 3}\left\{2 a_{n} \cos \theta_{n}\right\}+D C T_{N / 3}\left\{\sqrt{3} b_{n}^{\prime} \sin \theta_{n}-b_{n}^{\prime} \cos \theta_{n}\right\}\right. \\
& \left.+D C T_{N / 3}\left\{-\sqrt{3} c_{n} \sin \theta_{n}-c_{n} \cos \theta_{n}\right\}\right\} \\
& =\sqrt{\frac{1}{3} \frac{1}{\varepsilon_{k}}}-D C T_{N / 3}\left\{\left(2 a_{n}-a_{n}\right) \cos \theta_{n}+\sqrt{3} e_{n} \sin \theta_{n}\right\} \\
& =\sqrt{\frac{1}{3}} \frac{1}{\varepsilon_{k}} D C T_{N / 3}\left\{I D C T_{N / 3}\left\{2 A_{k}-D_{t}\right\} \cos \theta_{n}+I D C T_{N / 3}\left\{E_{k}\right\} \sqrt{3} \sin \theta_{n}\right\}
\end{aligned}
$$




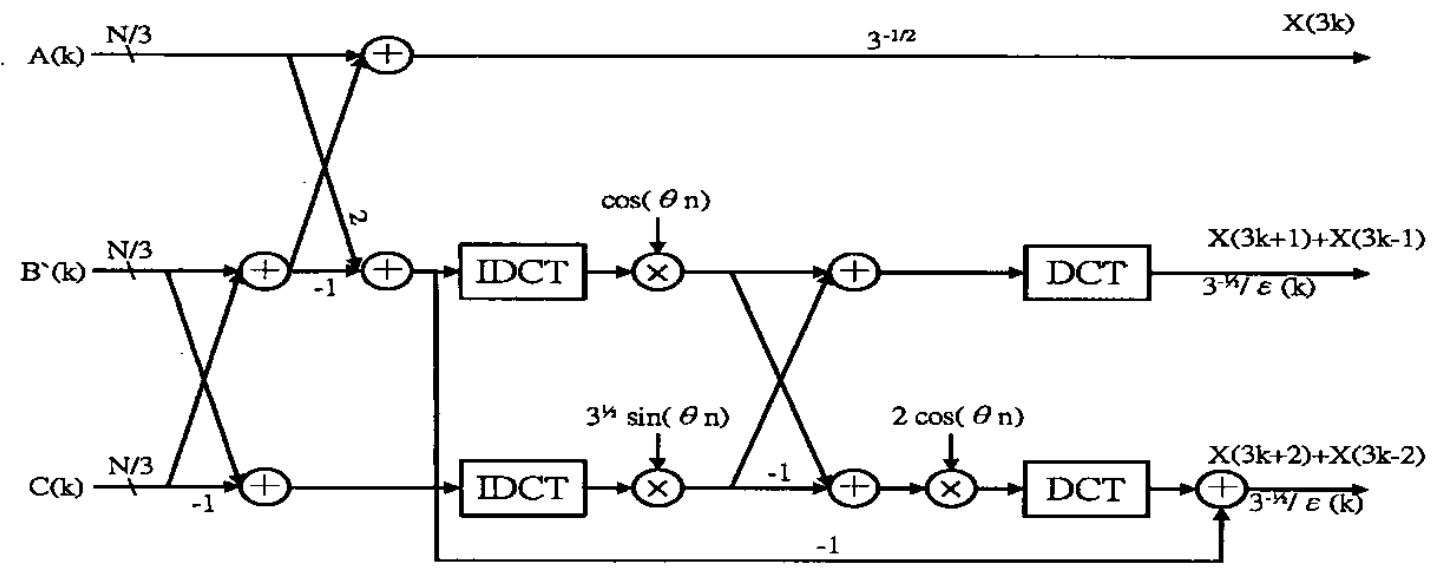

Iig. 2 Flow graph of the proposed algorithm

where

$$
\begin{aligned}
& d_{n}=b_{n}^{\prime}+c_{n} \\
& e_{n}=b_{n}^{\prime}-c_{n}
\end{aligned}
$$

As a consequence,

$$
\begin{aligned}
& D_{k}=(-1)^{k} B_{k}+C_{k} \\
& E_{k}=(-1)^{k} B_{k}-C_{k}
\end{aligned}
$$

\section{3. $X_{3 k+2}+X_{3 k-2}$ COEFFICIENTS CALCULATION}

The derivation of $X_{3 k+2}+X_{3 k-2}$ is similar to that of $X_{3 k+1}+X_{3 k-1}$. Therefore, we summarize the results as follows,

$$
\begin{aligned}
X_{3 k+2}+X_{3 k-2}= & \sqrt{\frac{1}{3}} \frac{1}{\varepsilon_{k}} D C T_{N / 3}\left\{I D C T_{N / 3}\left\{2 A_{k}-D_{k}\right\} \cos 2 \theta_{n}\right. \\
& \left.-I D C T_{N / 3}\left\{E_{k}\right\} \sqrt{3} \sin 2 \theta_{n}\right\}
\end{aligned}
$$

Further more,

$$
\begin{aligned}
\cos 2 \theta_{n} & =\cos \left(\theta_{n}+\theta_{n}\right)=2 \cos ^{2} \theta_{n}-1 \\
\sin 2 \theta_{n} & =\sin \left(\theta_{n}+\theta_{n}\right)=2 \cos \theta_{n} \sin \theta_{n}
\end{aligned}
$$

$$
\begin{aligned}
& X_{3 k+2}+X_{3 k-2} \\
&= \sqrt{\frac{1}{3} \frac{1}{\varepsilon_{k}}} D C T_{N / 3}\left\{I D C T_{N / 3}\left\{2 A_{k}-D_{k}\right\}\left(2 \cos ^{2} \theta_{n}-1\right)\right. \\
&-I D C T_{N / 3}\left\{E_{k}\left\{\sqrt{3}\left(2 \cos \theta_{n} \sin \theta_{n}\right)\right\}\right. \\
&= \sqrt{\frac{1}{3}} \frac{1}{\varepsilon_{k}}\left\{D C T _ { N / 3 } \left\{2 \operatorname { c o s } \theta _ { n } \left\{I D C T_{N / 3}\left\{2 A_{k}-D_{k}\right\} \cos \theta_{n}\right.\right.\right. \\
&\left.\left.\left.-I D C T_{N / 3}\left\{E_{k}\right\} \sqrt{3} \sin \theta_{n}\right\}\right\}-\left(2 A_{k}-D_{k}\right)\right\}
\end{aligned}
$$

According to the derivation above, we can construct the entire structure in Fig.2.

\section{4. $X_{3 k+1}, X_{3 k+2}$ COEFFICIENTS CALCULATION}

Since we have obtained $X_{3 k+1}+X_{3 k-1}$ and $X_{3 k+2}+H_{3 k-2}$, the next question is how to calculate $X_{3 k+1}$ and $X_{3 k+2}$ from them. According to (1), $x_{k}$ is an even function of $\mathrm{k}$ because both $\varepsilon_{k}$ and $\cos \{(2 n+1) k \pi / 2 N\}$ are even functions of $\mathrm{k}$. Therefore, for $k=0$,

$$
\begin{aligned}
& X_{3 k+1}+\left.X_{3 k-1}\right|_{k=0}=X_{1}+X_{-1}=2 X_{1} \\
& X_{3 k+2}+\left.X_{3 k-2}\right|_{k=0}=X_{2}+X_{-2}=2 X_{2}
\end{aligned}
$$

Using sequences $X_{3 k+1}+X_{3 k-1}$ and $X_{3 k+2}+X_{3 k-2}$ together with the initial conditions $X_{1}$ and $X_{2}$, we can derive $X_{3 k+1}$ and $X_{3 k+2}$ in an iterative approach. The flow graph is illustrated in Fig. 3 where $N / 3$ is chosen to be 8 for example.

\section{COMPUTATIONAL COMPLEXITY}

From the proposed structures in Fig.2 and Fig.3, the computation of $X_{3 k}, X_{3 k+1}+X_{3 k-1}$ and $X_{3 k+2}+X_{3 k-2}$ requires 7 length- $N / 3$ vector additions, 3 length- $N / 3$ vector multiplications and 4 length- $N / 3$ DCTs, assuming that the DCT and the IDCT are of the same complexity. Furthermore, we need 2 more scalar multiplications to accomplish the scaling of $1 / \varepsilon_{k}$ in the last stage of Fig. 2. 


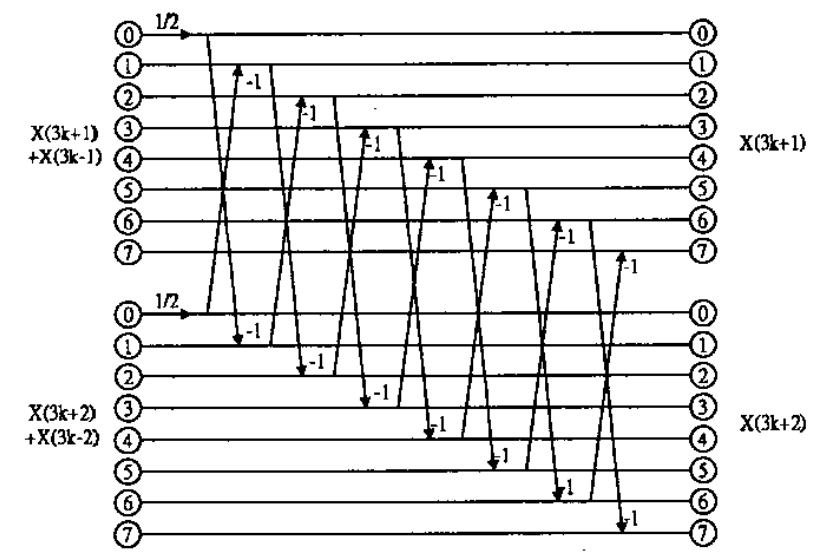

Fig. 3 Flow graph of $\mathrm{r}_{3 k+1}$ and $\mathrm{X}_{3 k+2}$

On the other hand, the computation of $X_{3 k+1}$ and $X_{3 k+2}$ in Fig. 3 requires 2 length- $(N / 3-1)$ vector additions. Since the scaling of $1 / 2$ in the first stage of Fig. 3 could be absorbed into the last stage of Fig. 2, there would be no more multiplications here.

For the purpose of simplicity, we choose $N=2^{m} \times 3$ in the following comparisons such that the fast $\mathrm{DCT}$ could be used to implement length- $N / 3 \mathrm{DC} T s$, where $m$ is a positive integer. According to [3], the arithmetic complexity of a length- $N / 3$ fast DCT is

$$
\begin{aligned}
M_{N / 3}^{D C T} & =M_{2^{m}}^{D C T}=(m / 2) \times(N / 3) \\
A_{N / 3}^{D C T} & =A_{2^{m}}^{D C T}=(3 m / 2) \times(N / 3)-(N / 3)+1
\end{aligned}
$$

The total numbers of multiplications $M_{N}^{P}$ and additions $A_{N}^{p}$ of the proposed structure then become

$M_{N}^{P}=3(N / 3)+2+4 M_{N / 3}^{D C T}=N(2 m / 3+1)+2$

$A_{N}^{P}=7(N / 3)+2(N / 3-1)+4 A_{N / 3}^{D C T}=N(2 m+5 / 3)+2$

As for the conventional approach, the complexity is equal to 3 length- $N / 3$ DCTs and 1 length- $N$ DCT. The total numbers of multiplications $M_{N}^{T}$ and additions $A_{N}^{T}$ of the conventional structure then become [2]

$$
\begin{aligned}
M_{N}^{T} & =3 \times M_{N / 3}^{D C T}+M_{N}^{D C T}=N(m+1)-2 \\
A_{N}^{T} & =3 \times A_{N / 3}^{D C T}+A_{N}^{D C T}=N(3 m+1)+1
\end{aligned}
$$

We summarize the computational complexity of these two approaches in Table 1. Note that the computational saving percentage increases with $N$. Moreover, for $N=8 \times 3$, which is the standard block dimension for image and video coding, about $30 \%$ operations are saved.

\begin{tabular}{|c|c|c|c|c|c|c|}
\hline \multirow{2}{*}{$N$} & Mul & Add & \multicolumn{2}{|c|}{ Conventional } & \multicolumn{2}{c|}{ Proposed } \\
\cline { 4 - 7 } & Save & Save & Mul & Add & Mul & Add \\
\hline 12 & $13 \%$ & $21 \%$ & 34 & 85 & 30 & 70 \\
\hline $\mathbf{2 4}$ & $\mathbf{2 7 \%}$ & $\mathbf{3 0} \%$ & $\mathbf{9 4}$ & $\mathbf{2 4 1}$ & $\mathbf{7 4}$ & $\mathbf{1 8 6}$ \\
\hline 48 & $34 \%$ & $34 \%$ & 238 & 625 & 178 & 466 \\
\hline 96 & $38 \%$ & $37 \%$ & 574 & 1537 & 417 & 1122 \\
\hline
\end{tabular}

Table 1 Comparison of two approaches

\section{CONCLUSIONS}

We propose an efficient approach to compute a length- $N$ DCT given three consecutive length- $N / 3$ DCTs. The operations could be saved up to $30 \%$ when the block size is chosen to be 8 , and the larger the block size is, the more operations we save. Another advantage is that only length- $N / 3$ DCT's and IDCTs are required instead of length- $N$ DCTs. Combining with Skodras's method, scaling a DCT-based image by the factor of $N / 2^{\alpha} 3^{\beta}$ is easily realizable by cascading $\alpha$ Skodras's structures and $\beta$ our structures. This provides more flexibility and efficiency in the application of fractional scaling, especially in the rising standard of HDTV whose screen size is usually set to be 16:9.

\section{REFERENCES}

[1] Athanassios N. Skodras, "Direct transform to transform computation," IEEE Signal Processing Letters, Volume: 6, Issue: 8, Aug. 1999, Pages: 202 204

[2] Yuk-Hee Chan and Wan-Chi Siu, "Fast radix-3/6 algorithms for the realization of the discrete cosine transform," IEEE International Symposium on Circuits and Systems, Volume: 1, 3-6 May 1992, Pages: 153 $156 \mathrm{vol} .1$

[3] C. W. Kok, "Fast algorithm for computing discrete cosine transform," IEEE Transactions on Signal Processing, Volume: 45, Issue: 3, March 1997, Pages: $757-760$

[4] HyunWook Park, YoungSeo Park, and Seung-Kyun $\mathrm{Oh}$, "L/M-fold image resizing in block-DCT domain using symmetric convolution," IEEE Transactions on Image Processing, Volume: 12, Issue: 9, Sept. 2003, Pages: 1016 - 1034

[5] Yunlong Zhao, M.S. Kankanhall, Tat-Seng Chua, "Fractional scaling of image and video in DCT domain," Proc. of IEEE International Conference on Image Processing, Barcelona, Spain, Volume: 1, Sept. 14-17, 2003, Pages: 185 - 188 